CANCER IMMUNOTHERAPY

\section{Copycat scaffolds induce T cell expansion}

Therapeutic ex vivo T cell expansion may be induced by a new scaffold system that mimics antigenpresenting cells (APCs), report David Mooney and colleagues in Nature Biotechnology.

T cell-based therapies have shown promise for the treatment of cancer, and involve isolating $\mathrm{T}$ cells from a patient and manipulating them ex vivo to induce the expansion of therapeutic $\mathrm{T}$ cells, which are then transferred back into the patient. Effective T cell expansion requires three signals, which in vivo are provided by APCs: stimulation of the $\mathrm{T}$ cell receptor; stimulation of the co-receptor CD28 and the provision of the pro-survival cytokine interleukin-2.

Ex vivo T cell expansion is challenging, as demonstrated by the limitations of the two major approaches: co-culture with autologous APCs or the use of commercially available beads (Dynabeads) bound to T cell activation cues that mimic APCs. "Isolating and culturing autologous APCs on a donor-specific basis increases the complexity and cost required to manufacture therapeutic T cells; furthermore, there can be high variability between donors," explains Mooney. "Dynabeads are convenient and reproducible, but present the cues that drive $\mathrm{T}$ cell expansion in an unnatural manner, which can lead to suboptimal T cell expansion rates and diminished $\mathrm{T}$ cell functionality. In addition, Dynabeads are non-degradable and can only present a limited set of signals."

The new system uses APCmimetic scaffolds (APC-ms), which are prepared from high-aspect ratio mesoporous silica micro-rods that are adsorbed with interleukin-2. Following this, the micro-rods are coated with liposomes, which contain biotinylated lipids, to form supported lipid bilayers. These supported lipid bilayers are loaded with membrane-bound $\mathrm{T}$ cell activation cues, such as anti-CD3 (CD3 is a component of the T cell receptor) and anti-CD28, using a streptavidin intermediate. In cell culture, the cue-loaded lipid bilayer-microrod composites form spontaneous 3D scaffolds, which present activation cues to infiltrating T cells whilst releasing adsorbed interleukin-2 in a sustained manner.
Compared with Dynabeads, APC-ms enabled a twofold to tenfold greater polyclonal expansion of primary mouse and human T cells. Furthermore, the type of $\mathrm{T}$ cell $\left(\mathrm{CD}^{+}\right.$ cytotoxic T cell or $\mathrm{CD}^{+}$helper T cell) that was induced to proliferate could be manipulated by modulating the formulation of APC-ms, which could be of clinical importance. Moreover, compared with co-culture with autologous APCs, APC-ms could induce a greater expansion of antigen-specific cytotoxic T cells isolated from mice or humans. Importantly, APC-msexpanded CD19 (a receptor found on B cells) chimeric antigen receptor T cells showed a similar efficacy to Dynabead-expanded cells in preventing tumour growth in an in vivo mouse xenograft lymphoma model.

"Our system allows a number of signals to be combined, is degradable and can be reproducibly manufactured," says Mooney. "In addition, APC-ms can present a variety of cues to $\mathrm{T}$ cells in a more physiologically relevant manner than the Dynabead system." In the future, the researchers envisage exploring how other cues impact the expansion of specific T cell populations and performing mechanistic studies to further define the potency of this system. "From a translational perspective, we hope to partner with relevant entities to move this system to expand $\mathrm{T}$ cells used to treat patients," concludes Mooney.

Shimona Starling

Associate Editor, Nature Reviews Cross-Journal Editorial Team

ORIGINAL ARTICLE Cheung, A. S. et al. Scaffolds that mimic antigen-presenting cells enable ex vivo expansion of primary T cells. Nat. Biotechnol. https://doi.org/10.1038/nbt.4047 (2018) 